\title{
Fauna parasitária dos híbridos siluriformes cachapinta e jundiara nos primeiros estágios de desenvolvimento
}

\author{
Arlene Sobrinho Ventura(1), Gabriela Tomas Jerônimo ${ }^{(2)}$, Eduardo Luiz Tavares Gonçalves (2), \\ Bianca Rafaela Fiori Tamporoski ${ }^{(3)}$, Maurício Laterça Martins ${ }^{(2)}$ e Márcia Mayumi Ishikawa ${ }^{(3)}$
}

\begin{abstract}
(1)Faculdade Anhanguera de Dourados, Rua Manoel Santiago, no 1.775, Vila São Luis, CEP 79925-150 Dourados, MS. E-mail: arlenesventura@yahoo.com.br (2)Universidade Federal de Santa Catarina, Departamento de Aquicultura, Laboratório Aquos Sanidade de Organismos Aquáticos, Rodovia Admar Gonzaga, no 1.346, CEP 88040-900 Florianópolis, SC. E-mail: gabrielatj@gmail.com, eltgoncalves@gmail.com, mlaterca@cca.ufsc.br ${ }^{(3)}$ Embrapa Agropecuária Oeste, BR-163, Km 253,6, CEP $79804-970$ Dourados, MS. E-mail: bibirafaft@yahoo.com.br, marcia.ishikawa@embrapa.br
\end{abstract}

Resumo - O objetivo deste trabalho foi avaliar a fauna parasitária dos peixes híbridos cachapinta (Pseudoplatystoma corruscans macho x P. reticulatum fêmea) e jundiara (Leiarius marmoratus macho x P. reticulatum fêmea) no período de larvicultura. Um total de 315 animais, 126 híbridos jundiara e 189 híbridos cachapinta, foram examinados de acordo com o estágio de desenvolvimento: larvas, pós-larvas e alevinos. Larvas e pós-larvas foram prensadas entre lâmina e lamínula e observadas sob microscopia óptica. Os alevinos foram observados externamente em estereomicroscópio e necropsiados para avaliação interna dos órgãos. Foram aferidos os parâmetros de qualidade de água em todas as fases de cultivo. O exame parasitológico revelou a presença dos protozoários Epistylis sp. e Trichodina sp., no tegumento e nas brânquias, Ichthyophthirius multifiliis no tegumento, dos helmintos Monogenea nas brânquias, e das metacercárias de digenéticos em brânquias, baço, rins, fígado e intestino, em ambos os híbridos analisados. Os parasitos Epystilis sp., Trichodina sp. e metacercária de digenéticos ocorrem com maior frequência nos dois híbridos, nas fases de pós-larvas e alevinos. Há maior prevalência e diversidade de parasitos na fase de alevinagem dos híbridos estudados.

Termos para indexação: Epistylis, Ichthyophthirius multifiliis, Trichodina, bagre, larvicultura, parasitos.

\section{Parasitic fauna of the siluriform hybrids cachapinta and jundiara in the first stages of development}

\begin{abstract}
The objective of this work was to evaluate the parasitic fauna of the hybrid fish cachapinta (Pseudoplatystoma corruscans male $\mathrm{x}$ P. reticulatum female) and jundiara (Leiarius marmoratus male $\mathrm{x}$ P. reticulatum female) at the hatchery stages. A total of 315 animals, 126 hybrids of jundiara and 189 hybrids of cachapinta were examined according to their development stage: larvae, post-larvae, and fingerlings. Larvae and post-larvae were pressed between a slide and a cover slip and were observed under optical microscopy. Fingerlings were externally observed in a stereomicroscope and dissected for internal evaluation of organs. Water quality parameters were measured in all cultivation phases. The parasitological exam showed the presence of the protozoa Epistylis sp. and Trichodina sp., on integument and gills, and Ichthyophthirius multifiliis on integument, of the helminth Monogenea on gills, and of digenean metacercariae on gills, spleen, kidneys, liver, and intestines in both hybrid fish evaluated. The parasites Epistylis sp., Trichodina sp., and digenean metacercariae occur most frequently in the two hybrids at the stages of post-larvae and fingerlings. There is a higher prevalence and diversity of parasites in the fingerling phase of the studied hybrids.
\end{abstract}

Index terms: Epistylis, Ichthyophthirius multifiliis, Trichodina, catfish, hatchery, parasite.

\section{Introdução}

Os bagres da família Pimelodidae são apreciados por sua esportividade para a pesca e se destacam na produção intensiva, em razão do alto potencial para exploração industrial, com eficiente conversão alimentar e alta taxa de crescimento (Rotta, 2003a). Além disso, apresentam características organolépticas e rendimento de carcaça favorável ao atendimento do mercado consumidor (Crepaldi et al., 2006; Inoue et al., 2009).

Recentemente, a criação de peixes híbridos vem ganhando espaço nos sistemas de cultivo do Brasil central, em virtude da grande aceitabilidade nos mercados nacional e internacional (Pádua et al., 2012). Entre os híbridos mais produzidos estão o cachapinta 
ou surubim híbrido, resultante do cruzamento entre a fêmea de cachara (Pseudoplatystoma reticulatum) e o macho de pintado ( $P$. corruscans); e o híbrido jundiara, resultante do cruzamento do macho de jundiá-da-amazônia (Leiarus marmoratus) e a fêmea de cachara ( $P$. reticulatum).

Embora as técnicas de reprodução artificial em peixes já estejam avançadas para algumas espécies nativas (Zaniboni-Filho \& Nuñer, 2004), ainda são escassas as informações que permitem expandir a produção de larvas de peixes híbridos para suprir a demanda do mercado. Na etapa de larvicultura, os peixes apresentam-se sensíveis ao estresse e às variações bruscas na qualidade de água (Inoue et al., 2009), o que os torna susceptíveis a agentes parasitários, uma vez que o manejo sanitário ineficiente resulta em perdas de inúmeros lotes por enfermidades.

Há conhecimento de poucos trabalhos que relatam a fauna parasitária nas fases iniciais de peixes de água doce no Brasil e no mundo. Pode \& Dick (1985) encontraram seis tipos de parasitos, entre eles Cestoda, Monogenea, Digenea, Acanthocephala, Nematoda e copépodes de crustáceos parasitos em larvas de Stizostedion vitreum no Canadá. Navrátil (1991) registrou parasitismo em larvas e alevinos de Barbus barbus, Chondrostoma nasus, Leuciscus cephalus e Aspius aspius. Bhuiyan \& Musa (2008) estudaram a prevalência sazonal e a intensidade de infestação de ectoparasitos em alevinos de carpas (Cyprinus carpio) em Bangladesh. Por sua vez, Hossain et al. (2008) constataram a prevalência de ectoparasitos em alevinos de Hypophthalmichthys molitrix, Ctenopharyngodon idella, C. carpio, Puntius gonionotus, Catla catla, Labeo rohita, Cirrhina mrigala, no Distrito de Bogra, Blangladesh. Thien et al. (2009) avaliaram a ocorrência de parasitos digenéticos em larvas e juvenis de 15 peixes de água doce no Vietnã, entre eles o tambaqui (Colossoma macropomum), peixe nativo do Brasil. Majoros (1999) verificou a ocorrência de metacercárias de Diplostomum sp. em larvas de C. carpio.

No Brasil, Andrade et al. (2001) observaram digenéticos, nematoides, protozoários, Monogenea, cestoides e acantocéfalos em alevinos de matrinxã (Brycon cephalus) coletados em ambiente natural, na Amazônia Central. Araújo et al. (2009) avaliaram as infecções parasitárias no período de alevinagem de pirarucu (Arapaima gigas), na Amazônia Central, e registraram $36,76 \%$ de mortalidade causada por
Dawestrema sp. (Monogenea) e Trichodina sp. Efeitos negativos na produção de alevinos de surubim híbrido (P. corruscans $\mathrm{x}$ Pseudoplatystoma reticulatum) foram observados em animais parasitados por Ichthyophthirius multifiliis (Ishikawa et al., 2011), bem como os protozoários Epistylis sp. e Trichodina sp. (Pádua et al., 2012).

Portanto, é relevante investigar os parasitos que acometem os peixes durante o período de larvicultura e alevinagem, a fim de estabelecer medidas profiláticas adequadas para controlar as perdas de lotes e evitar a disseminação para as demais fases de produção.

O objetivo deste trabalho foi avaliar a fauna parasitária dos peixes híbridos, cachapinta e jundiara, no período de larvicultura.

\section{Material e Métodos}

O experimento foi realizado em uma unidade produtora de alevinos, no Município de Dourados, MS $\left(22^{\circ} 19^{\prime} 43^{\prime \prime} \mathrm{S}, 54^{\circ} 43^{\prime} 55^{\prime \prime} \mathrm{W}\right)$. Os peixes foram divididos em fases de desenvolvimento segundo Rotta (2003b): larvas, recém-eclodidos que ainda não ingeriram alimento exógeno; pós-larvas, que, após a absorção do saco vitelínico, ingeriram alimento exógeno; e alevinos, que já apresentam características do peixe adulto com 4,01 $\pm 1,59 \mathrm{~g}$ de massa corpórea média.

Foram avaliados 315 peixes híbridos nas fases iniciais de larvicultura, sendo 126 de híbrido jundiara (L. marmoratus macho x $P$. reticulatum fêmea) (53 larvas, 53 pós-larvas e 20 alevinos) e 189 de híbrido cachapinta ( $P$. corruscans macho $\mathrm{x} P$. reticulatum fêmea) (62 larvas, 69 pós-larvas e 58 alevinos).

Os animais estavam estocados em diferentes locais de acordo com a sua classificação. Para ambas as espécies, as larvas eram acomodadas em incubadoras, e as pós-larvas, em caixas de cimento. Já os alevinos do híbrido cachapinta eram estocados em tanques-rede, e os híbridos de jundiara, em viveiro escavado.

Para a análise parasitológica, as larvas e as pós-larvas foram capturadas com auxílio de puçás, prensadas entre lâmina/lamínula e submetidas à observação em microscópio para detecção e quantificação dos parasitos. Os alevinos foram capturados com rede de arrasto, sacrificados por aprofundamento anestésico em solução de óleo de cravo (70 $\left.\mathrm{mg} \mathrm{L}^{-1}\right)$ e observados em estereomicroscópio para avaliação do tegumento e posterior necropsia para observação macroscópica 
da cavidade e dos órgãos (Comitê de Ética 23080.0 29979/2009-05/CEUA/UFSC). Em seguida, foram feitos prensados entre lâmina/lamínula dos órgãos para exame microscópico.

Os parasitos foram identificados segundo Thatcher (2006) e Martins et al. (2010). A partir dos resultados obtidos, foram calculadas a taxa de prevalência, a intensidade média e a abundância média para cada parasito conforme Bush et al. (1997): taxa de prevalência $=$ (número de peixes infectados/número de peixes analisados) $\times 100$; intensidade média $=$ número total de uma espécie de parasito na amostra/número de peixes infectados por aquele parasito; e abundância média $=$ número total de uma espécie de parasito na amostra/número de peixes examinados (infectados e não infectados).

Diariamente, os parâmetros aquáticos, como oxigênio dissolvido e temperatura da água, foram medidos com oxímetro digital YSI 550, (YSI Incorporated, Yellow Springs, OH, EUA), e o potencial hidrogeniônico foi determinado com pHmetro portátil YSI PH10, (YSI Incorporated, Yellow Springs, OH, EUA). Amostras da saída de água foram coletadas para dosagem de amônia total, nitrito, nitrato, ortofosfato, ferro total e alcalinidade, por meio de kit colorimétrico (Alfakit, Florianópolis, SC). Os resultados obtidos foram submetidos à análise descritiva.

Os valores de contagem dos parasitos foram transformados em $(\mathrm{Y}+0,5)^{1 / 2}$, em razão da baixa prevalência. Em seguida, foi aplicada análise de variância fatorial, com auxílio do programa Statistica 8.0, para comparar as espécies e os estádios dos peixes. A homogeneidade das variâncias foi testada pelo teste de Barlett, e as médias foram comparadas pelo teste de Tukey, a 5\% de probabilidade (ZAR, 1999).

\section{Resultados e Discussão}

Os valores de $\mathrm{pH}$ e temperatura se mantiveram semelhantes nos ambientes de cultivo para todas as classes de ambas as espécies estudadas. Os demais parâmetros apresentaram variações bruscas para pós-larvas do híbrido jundiara, estocado sob alta densidade de aproximadamente mil peixes em 0.5 ha de lâmina d'agua, em tanque escavado, e alimentado com alimento natural. A diminuição do oxigênio dissolvido para pós-larvas do híbrido cachapinta também pode ser atribuída à elevada densidade de estocagem em caixas de cimento (Tabela 1). Parâmetros de ferro total, nitrato, nitrito e ortofosfato apresentaram elevados desvios-padrão, pois os animais foram coletados de diferentes ambientes de criação, sob diferentes níveis de arraçoamento, tendo diferido quanto às concentrações de compostos excretados.

O exame parasitológico dos dois híbridos revelou a presença dos protozoários Epistylis sp. e Trichodina sp. no muco da superfície corporal e nas brânquias, e Ichthyophthirius multifiliis no muco; dos helmintos Monogenea nas brânquias; e das metacercárias de digenéticos encistadas em brânquias, baço, rins, fígado e parede intestinal (Tabela 2).

A prevalência de Epistylis sp. foi significativamente maior no muco de alevinos do que em larvas e pós-larvas de ambas as espécies (Tabela 2). Porém, no híbrido cachapinta, sua prevalência foi maior do que no híbrido jundiara. Não foi possível quantificar estes parasitos, pois apresentam forma colonial.

O peritríquio Epistylis sp. utiliza seus hospedeiros como substrato de fixação e transporte, o que agrava lesões pré-existentes em tegumento e brânquias e causa alterações nas trocas gasosas (Eiras et al., 2010). Em estudo na República Tcheca, Navrátil (1991) relatou $E$. lwoffi como parasito de juvenis de B. barbus, C. nasus e A.aspius, com prevalências de 10, 45 e 25\%, respectivamente. No presente trabalho, a prevalência foi maior para alevinos de jundiara, com $45,3 \%$ das pós-larvas e 95\% de alevinos parasitados. Já para o híbrido cachapinta, a prevalência foi de 18,9\% apenas para alevinos (Tabela 2). Estes resultados corroboram os de Pádua et al. (2013), que observaram maior prevalência no híbrido jundiara e menor prevalência em cachapinta, também em alevinos. Portanto, é possível inferir que o híbrido jundiara pode ser mais susceptível a esse peritríquio do que o cachapinta.

Os tricodinídeos foram ausentes somente em larvas do híbrido jundiara e apresentaram maiores valores de intensidade média em pós-larvas. Já em alevinos de híbrido cachapinta, a intensidade média e a abundância média foram maiores do que nas demais fases (Tabela 3). Entre as espécies, estes parasitos foram observados com maior frequência no muco dos alevinos de cachapinta do que no do híbrido jundiara.

Em condições favoráveis, estes parasitos provocam lesões nas brânquias de seus hospedeiros e podem levar a morte (Pádua et al., 2012). Os valores de 
prevalência de Trichodina sp., obtidos tanto para alevinos do híbrido jundiara quanto para os do híbrido cachapinta, foram maiores que os relatados por
Pádua et al. (2012) em alevinos de surubim híbrido (P. reticulatum $\mathrm{x}$ P. corruscans). Martins et al. (2010), ao avaliar o bagre Ictalurus punctatus, observaram

Tabela 1. Parâmetros de qualidade de água em sistema de cultivo de híbridos de jundiara (Leiarius marmoratus macho $\mathrm{x}$ Pseudoplatystoma reticulatum fêmea) e cachapinta ( $P$. corruscans macho x P. reticulatum fêmea), em diferentes fases de desenvolvimento.

\begin{tabular}{|c|c|c|c|c|c|c|}
\hline \multirow[t]{2}{*}{ Parâmetros } & \multicolumn{3}{|c|}{ Jundiara } & \multicolumn{3}{|c|}{ Cachapinta } \\
\hline & Larva & Pós-larva & Alevino & Larva & Pós-larva & Alevino \\
\hline $\mathrm{pH}$ & $7,13 \pm 0,18$ & $6,76 \pm 0,28$ & $6,72 \pm 0,37$ & $7,19 \pm 0,25$ & $6,66 \pm 0,01$ & $6,98 \pm 0,33$ \\
\hline Oxigênio (mg L-1) & $5,63 \pm 1,15$ & $2,66 \pm 0,97$ & $5,03 \pm 1,31$ & $3,78 \pm 0,14$ & $1,92 \pm 0,11$ & $4,34 \pm 1,04$ \\
\hline Temperatura $\left({ }^{\circ} \mathrm{C}\right)$ & $26,95 \pm 2,50$ & $27,63 \pm 1,17$ & $27,15 \pm 0,17$ & $28,00 \pm 0,00$ & $28,95 \pm 0,49$ & $28,40 \pm 0,97$ \\
\hline Alcalinidade $\left(\mathrm{mg} \mathrm{L}^{-1}\right)$ & $26,25 \pm 2,50$ & $45,0 \pm 23,09$ & $27,50 \pm 2,89$ & $27,50 \pm 3,54$ & $25,00 \pm 0,00$ & $24,29 \pm 3,96$ \\
\hline Amônia (mg L'-1) & $0,10 \pm 0,09$ & $0,60 \pm 0,70$ & $0,33 \pm 0,32$ & $0,30 \pm 0,43$ & $0,30 \pm 0,09$ & $0,15 \pm 0,07$ \\
\hline Ferro total $\left(\mathrm{mg} \mathrm{L}^{-1}\right)$ & $0,14 \pm 0,10$ & $0,31 \pm 0,24$ & $0,14 \pm 0,10$ & $0,13 \pm 0,18$ & $0,13 \pm 0,18$ & $0,03 \pm 0,07$ \\
\hline Nitrato $\left(\mathrm{mg} \mathrm{L}^{-1}\right)$ & $0,33 \pm 0,66$ & $3,88 \pm 1,11$ & $2,11 \pm 2,44$ & $4,43 \pm 0,00$ & $4,43 \pm 0,00$ & $2,19 \pm 2,28$ \\
\hline Nitrito $\left(\mathrm{mg} \mathrm{L}^{-1}\right)$ & $0,00 \pm 0,00$ & $0,03 \pm 0,00$ & $0,02 \pm 0,02$ & $0,03 \pm 0,00$ & $0,03 \pm 0,00$ & $0,02 \pm 0,02$ \\
\hline Ortofosfato (mg L-1) & $0,81 \pm 0,13$ & $1,19 \pm 0,38$ & $0,56 \pm 0,38$ & $0,38 \pm 0,53$ & $0,88 \pm 0,18$ & $0,38 \pm 0,42$ \\
\hline
\end{tabular}

Tabela 2. Prevalência (\%) e sítio de infecção de parasitas em híbridos de jundiara (Leiarius marmoratus macho $\mathrm{x}$ Pseudoplatystoma reticulatum fêmea) e cachapinta (P. corruscans macho x P. reticulatum fêmea), em diferentes fases de desenvolvimento ${ }^{(1)}$.

\begin{tabular}{|c|c|c|c|c|c|c|c|}
\hline \multirow[t]{2}{*}{ Parasitos } & \multicolumn{3}{|c|}{ Jundiara } & \multicolumn{3}{|c|}{ Cachapinta } & \multirow[t]{2}{*}{ Sítio de infecção } \\
\hline & $\begin{array}{c}\text { Larva } \\
(n=53)\end{array}$ & $\begin{array}{c}\text { Pós-larva } \\
(\mathrm{n}=53)\end{array}$ & $\begin{array}{l}\text { Alevino } \\
(n=20)\end{array}$ & $\begin{array}{c}\text { Larva } \\
(n=62)\end{array}$ & $\begin{array}{c}\text { Pós-larva } \\
(\mathrm{n}=69)\end{array}$ & $\begin{array}{c}\text { Alevino } \\
(\mathrm{n}=58)\end{array}$ & \\
\hline Epistylis sp. & $0 \mathrm{c}$ & $45,3 b$ & $95 \mathrm{a}$ & $0 \mathrm{~b}$ & $\mathrm{Ob}$ & $18,9 \mathrm{a}$ & Muco e brânquias \\
\hline Trichodina sp. & 0 & 11,3 & 50 & 1,6 & 26,1 & 31 & Muco e brânquias \\
\hline Ichthyophthirius multifiliis & 0 & 1,9 & 0 & 0 & 0 & 0 & Muco \\
\hline Monogenea & 0 & 0 & 0 & 0 & 1,6 & 1,7 & Brânquias \\
\hline Metacercárias & 0 & 7,5 & 50 & 0 & 10,1 & 86,2 & Brânquias, baço, rins, fígado, intestino \\
\hline
\end{tabular}

${ }^{(1)}$ Médias seguidas de letras iguais, nas linhas, não diferem pelo teste de Tukey, a 5\% de probabilidade.

Tabela 3. Média \pm desvio-padrão da intensidade e da abundância de parasitas em híbridos de jundiara (Leiarius marmoratus macho x Pseudoplatystoma reticulatum fêmea) e cachapinta (P. corruscans macho x $P$. reticulatum fêmea) nas diferentes fases de desenvolvimento ${ }^{(1)}$.

\begin{tabular}{|c|c|c|c|c|c|c|}
\hline \multirow[t]{2}{*}{ Parasitos } & \multicolumn{3}{|c|}{ Jundiara } & \multicolumn{3}{|c|}{ Cachapinta } \\
\hline & $\begin{array}{l}\text { Larva } \\
(\mathrm{n}=53)\end{array}$ & $\begin{array}{l}\text { Pós-larva } \\
(\mathrm{n}=53)\end{array}$ & $\begin{array}{l}\text { Alevino } \\
(\mathrm{n}=20)\end{array}$ & $\begin{array}{l}\text { Larva } \\
(\mathrm{n}=62)\end{array}$ & $\begin{array}{c}\text { Pós-larva } \\
(n=69)\end{array}$ & $\begin{array}{l}\text { Alevino } \\
(\mathrm{n}=58)\end{array}$ \\
\hline & \multicolumn{6}{|c|}{ Intensidade média } \\
\hline Epistylis sp. & - & - & - & - & - & - \\
\hline Trichodina sp. & $*$ & $11,5 \pm 14,4$ & $1,8 \pm 1,0$ & $1,0 \pm 0 \mathrm{~b}$ & $4,7 \pm 5,6 b$ & $59,0 \pm 74,5 \mathrm{a}$ \\
\hline Ichthyophthirius multifiliis & $*$ & $2,0 \pm 0$ & $*$ & $*$ & * & $*$ \\
\hline Monogenea & $*$ & * & $*$ & $*$ & $1,0 \pm 0$ & $4,0 \pm 0$ \\
\hline \multirow[t]{2}{*}{ Metacercárias } & $*$ & $2,7 \pm 2,1 b$ & $19,9 \pm 26,8 \mathrm{a}$ & $0 \mathrm{~b}$ & $7,7 \pm 10,3 b$ & $124,3 \pm 120,9 a$ \\
\hline & \multicolumn{6}{|c|}{ Abundância média } \\
\hline Epistylis sp. & - & - & - & - & - & - \\
\hline Trichodina sp. & $*$ & $1,3 \pm 14,4$ & $0,9 \pm 1,0$ & $0,01 \pm 0 \mathrm{~b}$ & $1,2 \pm 5,6 b$ & $18,3 \pm 74,54 a$ \\
\hline Ichthyophthirius multifiliis & $*$ & $0,03 \pm 0$ & $*$ & $*$ & $*$ & $*$ \\
\hline Monogenea & $*$ & $*$ & $*$ & $*$ & $0,01 \pm 0$ & $0,06 \pm 0$ \\
\hline Metacercárias & $*$ & $0,2 \pm 2,0 \mathrm{~b}$ & $9,9 \pm 26,8 \mathrm{a}$ & $*$ & $0,8 \pm 10,2 b$ & $107,2 \pm 120,9 a$ \\
\hline
\end{tabular}

${ }^{(1)}$ Médias seguidas de letras iguais, nas linhas, não diferem pelo teste de Tukey, a 5\% de probabilidade. (-) indica que o parasita não foi quantificado em razão da sua forma colonial. (*) indica que o parasita foi quantificado, mas que sua presença não foi detectada. 
prevalência de $100 \%$ de parasitos tricodinídeos. Estes parasitos são comuns em ambientes que favorecem seu desenvolvimento, como água de má qualidade (Hossain et al. 2008). $\mathrm{Na}$ fase de alevinagem, o acúmulo de matéria orgânica decorrente das sobras de ração, das cascas de ovos de náuplios de Artemia sp. e dos animais mortos no ambiente de cultivo fornece ambiente propício para o desenvolvimento de organismos patogênicos (Pavanelli et al., 2006; Inoue et al., 2009). Isso pode explicar a presença deste parasito com maior frequência em todas as fases de alevinagem no presente trabalho.

Metacercárias de digenéticos foram observadas em brânquias, baço, rins, fígado e intestino de alevinos dos híbridos. Resultado semelhante foi verificado em pós-larvas; porém, não foi possível a identificação do sítio de infecção já que os indivíduos foram prensados inteiros sobre lâmina/lamínula. Maior prevalência de digenéticos foi constatada em alevinos do híbrido cachapinta $(86,9 \%)$ do que nos do híbrido jundiara (50\%). Em pós-larvas, também houve maior parasitismo no híbrido cachapinta $(10,1 \%)$ do que no híbrido jundiara (7,5\%) (Tabela 2). Em larvas de ambos os híbridos, não foi observada a presença deste parasito. Entre os híbridos, houve maior parasitismo por metacercárias em alevinos de cachapinta.

Corrêa \& Brasil-Sato (2008) descreveram a ocorrência dos digenéticos Witenbergia witenbergi, Tylodelphys sp., Acanthostomum gnerii e Acanthostomum sp. em pintado (P. corruscans) oriundo do Rio São Francisco, com menor prevalência do que a observada no presente trabalho. Da mesma forma, Jerônimo et al. (2013) constataram menor ocorrência de metacercárias de digenéticos em cachara pantaneira ( $P$. reticulatum) mantido em baixa densidade de estocagem em tanques-rede, no Rio Paraguai. Isso fortalece a hipótese de que peixes menores são mais susceptíveis a digenéticos em razão da sua estratégia parasitária (Luque \& Poulin, 2004). Estes parasitos podem causar obstrução, necrose tecidual, dificuldade respiratória e catarata, e os sinais clínicos dependem da intensidade parasitária e do órgão acometido (Thatcher, 2006). A baixa infecção de digenéticos observada nas fases iniciais pode ser explicada pelo fato de as larvas e as pós-larvas terem sido mantidas em incubadoras e tanques de cimento com maior controle de qualidade ambiental, enquanto os alevinos foram mantidos em tanques-rede $\mathrm{e}$ viveiros escavados, ambientes onde havia contato direto com aves e moluscos, vetores desta parasitose.

Ichthyophthirius multifiliis foi observado apenas no muco de pós-larvas do híbrido jundiara com prevalências e intensidades médias menores do que as observadas por Navrátil (1991) nos ciprinídeos B. barbus, C. nasus, L. cephalus e A. aspius, os quais apresentaram taxa de prevalência entre 10 e $40 \%$. A temperatura é um dos fatores que afeta o desenvolvimento deste parasito (Ghiraldelli et al., 2007), o que permite concluir que este parasito é um dos principais limitantes para o cultivo de Rhamdia quelen no Sul do Brasil (Carneiro et al., 2005). Com temperaturas mais elevadas, Araújo et al. (2009) não observaram a ocorrência deste protozoário em alevinos de pirarucu, peixe oriundo de águas de temperaturas acima de $28^{\circ} \mathrm{C}$. Assim, a baixa prevalência encontrada no presente trabalho pode ser resultado de ambiente não propício para o desenvolvimento do parasito, com valores médios de temperatura de $26^{\circ} \mathrm{C}$.

Helmintos Monogenea foram observados apenas nas brânquias do híbrido cachapinta, não tendo diferido significativamente entre as fases de desenvolvimento. Em estudo realizado por Martins et al. (2002), os platelmintos Monogenea estão entre os principais agentes parasitários que acometem os peixes. Parasitos desta classe afetam principalmente as brânquias e podem causar hiperplasia e telangiectasia dos filamentos branquiais, o que dificulta as trocas gasosas (Pavanelli et al., 2006). Jerônimo et al. (2013) constataram prevalência de $80 \%$ de helmintos Monogenea em P. reticulatum cultivado em tanque-rede. Por sua vez, Campos et al. (2008) observaram 100\% de prevalência em cacharas ( $P$. fasciatum) de ambiente natural, no Rio Aquidauana. Esses resultados diferem dos obtidos no presente trabalho, em que a prevalência foi baixa, e as pós-larvas e os alevinos do híbrido cachapinta, os mais parasitados.

Em linhas gerais, os resultados obtidos podem contribuir para o conhecimento da fauna parasitária de híbridos resultantes do cruzamento de bagres da família Pimelodidae, em suas fases iniciais de criação, e também podem auxiliar as unidades produtoras de alevinos a desenvolverem controle sanitário sobre o processo produtivo, para manter a higidez dos alevinos comercializados.

Pesq. agropec. bras., Brasília, v.48, n.8, p.943-949, ago. 2013 DOI: 10.1590/S0100-204X2013000800019 


\section{Conclusões}

1. Os parasitos Epistylis sp., Trichodina sp. e metacercárias de digenéticos ocorrem com maior prevalência, nos peixes híbridos cachapinta (Pseudoplatystoma corruscans macho x P. reticulatum fêmea) e jundiara (Leiarius marmoratus macho $\mathrm{x}$ P. reticulatum fêmea), nas fases de pós-larvas e alevinos.

2. A fase de alevinagem dos híbridos cachapinta e jundiara apresenta maior prevalência e diversidade de parasitos.

\section{Agradecimentos}

Ao Conselho Nacional de Desenvolvimento Científico e Tecnológico (CNPq), pela concessão de bolsas; e ao Ministério da Pesca e Aquicultura (MPA) e à Embrapa Agropecuária Oeste (CPAO), pelo auxílio financeiro.

\section{Referências}

ANDRADE, S.M.S. de; MALTA, J.C. de O.; FERRAZ, E. Fauna parasitológica de alevinos de matrinchã Brycon cephalus (Gunther, 1869) coletados nos rio Negro e Solimões, na Amazônia Central. Acta Amazonica, v.31, p.263-273, 2001.

ARAÚJO, C.S.O.; GOMES, A.L.; TAVARES-DIAS, M.; ANDRADE, S.M.S.; BELEM-COSTA, A.; BORGES, J.T.; QUEIROZ, M.N.; BARBOSA, M. Parasitic infections in pirarucu fry, Arapaima gigas Schinz, 1822 (Arapaimatidae) kept in a semi-intensive fish farm in Central Amazon, Brazil. Veterinarski Archives, v.79, p.499-507, 2009.

BHUIYAN, A.S.; MUSA, A.S.M. Seasonal prevalence and intensity of infestation by the ectoparasites in carps relating to physico-chemical parameters in some ponds of Mymensingh and Bogra Districts of Bangladesh. Bangladesh Journal of Scientific and Industrial Research, v.43, p.411-418, 2008. DOI: 10.3329/ bjsir.v43i3.1158.

BUSH, A.O.; LAFFERTY, K.D.; LOTZ, J.M.; SHESTAK, A.W. Parasitology meets ecology on its own terms: Margolis et al. revisited. Journal of Parasitology, v.83, p.575-583, 1997. DOI: $10.2307 / 3284227$.

CAMPOS, C.M.; FONSECA, V.E.; TAKEMOTO, R.M.; MORAES, F.R. Fauna parasitária de cachara Pseudoplatystoma fasciatum (Siluriforme: Pimelodidae) do rio Aquidauana, Pantanal Sul Mato-grossense, Brasil. Acta Scientiarum. Biological Sciences, v.30, p.91-96, 2008. DOI: 10.4025/actascibiolsci. v30i1.1469.

CARNEIRO, P.C.F.; SCHORER, M.; MIKOS, J.D. Tratamentos terapêticos convencionais no controle do ectoparasita Ichthyophtirius multifiliis em jundiá (Rhamdia quelen). Pesquisa Agropecuária Brasileira, v.40, p.99-102, 2005. DOI: 10.1590/ S0100-204X2005000100015.
CORRÊA, R. de F. da S.S.; BRASIL-SATO, M. de C. Digenea in the surubim Pseudoplatystoma corruscans (Spix and Agassiz, 1829) (Siluriformes: Pimelodidae) of the Upper São Francisco River, State of Minas Gerais, Brazil. Brazilian Archives of Biology and Technology, v.51, p.1181-1185, 2008. DOI: 10.1590/ S1516-89132008000600013.

CREPALDI, D.V.; FARIA, P.M.C.; TEIXEIRA, E. de A.; RIBEIRO, L.P.; COSTA, Â.A.P.; MELO, D.C. de; CINTRA, A.P.R.; PRADO, S. de A.; COSTA, F.A.A.; DRUMOND, M.L.; LOPES, V.E.; MORAES, V.E. de. O surubim na aquacultura do Brasil. Revista Brasileira de Reprodução Animal, v.30, p.150-158, 2006.

EIRAS, J.C.; TAKEMOTO, R.M.; PAVANELLI, G.C. Diversidade dos parasitas de peixes de água doce do Brasil. Maringá: Clichetec, 2010.

GHIRALDELLI, L.; ADAMANTE, W. de B.; MARTINS, M.L.; MOURIÑO, J.L.P.; STREIT, A.A.R.; BERESTINAS, A.C.; LOUREIRO, C.; FRANCISCO, C.J. Infecção com trofozoítos de Ichthyophthirius multifiliis (Ciliophora) em Poecilia vivipara (poeciliidae) como hospedeiro experimental. Ciência Animal Brasileira, v.8, p.105-110, 2007.

HOSSAIN, M.D.; HOSSAIN M.K.; RAHMAN, M.H.; AKTER, A.; KHANOM, D.A. Prevalence of ectoparasites of carp fingerlings at Santaher, Bogra. University Journal of Zoology, v.27, p.17-19, 2008. DOI:10.3329/ujzru.v27i0.1947.

INOUE, L.A.K.A.; HISANO, H.; ISHIKAWA, M.M.; ROTTA M.A.; SENHORINI, J.A. Princípios básicos para produção de alevinos de surubins (pintado e cachara). Dourados: Embrapa Agropecuária Oeste, 2009. 26p.

ISHIKAWA, M.M.; PADUA, S.B. de; VENTURA, A.S.; CAPECCI, R.; VENDRUSCULO, A.; CARRIJO-MAUAD, J.R. Infestação por ictio em surubim híbrido durante a fase inicial de criação. Dourados: Embrapa Agropecuária Oeste, 2011. 5p. (Embrapa Agropecuária Oeste. Comunicado técnico, 165).

JERÔNIMO, G.T.; VENTURA, A.S.; PÁDUA, S.B. de; SATAKE, F.; ISHIKAWA, M.M.; MARTINS, M.L. Parasitofauna de cachara cultivado em tanque-rede no Rio Paraguai. Pesquisa Agropecuária Brasileira, v.48, p.1163-1166, 2013. DOI: 10.1590/ S0100-204X2013000800054.

LUQUE, J.L.; POULIN, R. Use of fish as intermediate hosts by helminth parasites: a comparative analysis. Acta Parasitologica, v.49, p.353-361, 2004.

MAJOROS, G. Mortality of fish fry as a result of specific and specific cercarial invasion under experimental conditions. Acta Veterinaria Hungarica, v.47, p.433-450, 1999. DOI: 10.1556/ AVet.47.1999.4.4.

MARTINS, M.L.; MARCHIORI, N.; NUNES, G.; RODRIGUES, M.P. First record of Trichodina heterodentata (Ciliophora: Trichodinidae) from channel catfish, Ictalurus punctatus cultivated in Brazil. Brazilian Journal of Biology, v.70, p.637-644, 2010. DOI: $10.1590 /$ S1519-69842010000300022.

MARTINS, M.L.; ONAKA, E.M.; MORAES, F.R. de; BOZZO, F.R.; PAIVA, A. de M. e F.C.; GONÇALVES, A. Recent studies on parasitic infections of freshwater fish cultivated in the state of São Paulo, Brazil. Acta Scientiarum, v.24, p.981-985, 2002. 
NAVRÁTIL, S. Parasitoses in the fry of selected freshwater fish species under the conditions of stripping and rearing. Acta Veterinaria Brno, v.60, p.357-366, 1991. DOI: 10.2754/ avb199160040357.

PÁDUA, S.B. de; ISHIKAWA, M.M.; KASAI, R.Y.D.; JERÔNIMO, G.T.; CARRIJO-MAUAD, J.R. Parasitic infestations in hybrid surubim catfish fry (Pseudoplatystoma reticulatum $\mathrm{x}$ P. corruscans). Revista Brasileira de Medicina Veterinária, v.34, p.235-240, 2012. DOI: 10.1007/s00436-012-3069-5.

PÁDUA, S.B. de; ISHIKAWA, M.M.; VENTURA, A.S.; JERÔNIMO, G.T.; MARTINS, M.L.; TAVARES, L.E.R. Brazilian catfish parasitized by Epistylis sp. (Ciliophora, Epistylididae), with description of parasite intensity score. Parasitology Research, v.112, p.443-446, 2013. DOI: 10.1007/s00436-012-3069-5.

PAVANELLI, G.C.; EIRAS, J. da C.; TAKEMOTO, R.M. Doenças de peixes: profilaxia, diagnóstico e tratamento. 2.ed. Maringá: Ed. UEM, 2006. 303p.

PODE, B.C.; DICK, T.A. Parasite recruitment by stocked walleye, Stizostedion vitreum vitreum (Mitchill), fry in a small boreal lake in central Canada. Journal of Wildlife Diseases, v.21, p.371-376, 1985.
ROTTA, M.A. Aspectos gerais da fisiologia e estrutura do sistema digestivo dos peixes relacionados à piscicultura. Corumbá: Embrapa Pantanal, 2003a. 48p. (Embrapa Pantanal. Documentos, 53).

ROTTA, M.A. Ictiômetro para biometria de surubins (pintado e cachara). Corumbá: Embrapa Pantanal, 2003b. 4p. (Embrapa Pantanal. Comunicado técnico, 28).

THATCHER, V.E. Amazon fish parasites. $2^{\text {nd }}$ ed. Moscow: Pensoft, 2006. 508p.

THIEN, C.P.; DALSGAARD, A.; NHAN, N.T.; OLSEN, A.; MURRELL, K.D. Prevalence of zoonotic trematode parasites in fish fry and juveniles in fish farms of the Mekong Delta, Vietnam. Aquaculture, v.295, p.1-5, 2009. DOI: 10.1016/j. aquaculture.2009.06.033.

ZANIBONI FILHO, E.; NUÑER, A.P. de O. Reprodução de peixes migradores de água doce do Brasil. In: CYRINO, J.E.P.; URBINATI, E.C.; FRACALOSSI, D.M.; CASTAGNOLLI, N. (Ed.). Tópicos especiais em piscicultura de água doce tropical intensiva. São Paulo: TecArt, 2004. p.45-74.

ZAR, J.H. Biostatistical analysis. $4^{\text {th }}$ ed. New Jersey: Upper Saddle River, 1999. 662p.

Recebido em 23 de agosto de 2011 e aprovado em 8 de junho de 2013 